\title{
THE CROSSING NUMBER OF JOIN OF THE GENERALIZED PETERSEN GRAPH $P(3,1)$ WITH PATH AND CYCLE ${ }^{1}$
}

\author{
ZhangDong OUYANG \\ Department of Mathematics \\ Hunan First Normal University \\ Changsha 410205, P.R. China \\ e-mail: oymath@163.com \\ JING WANG \\ Department of Mathematics and Information Sciences \\ Changsha University \\ Changsha 410003, P.R. China \\ e-mail: wangjing1001@hotmail.com \\ AND \\ YUANQIU HUANG \\ Department of Mathematics \\ Hunan Normal University \\ Changsha 410081, P.R. China \\ e-mail: hyqq@hunnu.edu.cn
}

\begin{abstract}
There are only few results concerning the crossing numbers of join of some graphs. In this paper, the crossing numbers of join products for the generalized Petersen graph $P(3,1)$ with $n$ isolated vertices as well as with the path $P_{n}$ on $n$ vertices and with the cycle $C_{n}$ are determined.
\end{abstract}

Keywords: crossing number, drawing, join product, generalized Petersen graph.

2010 Mathematics Subject Classification: 05C10, 05C38.

\footnotetext{
${ }^{1}$ The work was supported by the National Natural Science Foundation of China (Nos. 11301169 \& 11371133), Hunan Provincial Natural Science Foundation of China (Nos. 13JJ4110 \& 14JJ3138) and Hunan Education Department Talented Foundation (No. 16B028).
} 


\section{REFERENCES}

[1] J.A. Bondy and U.S.R. Murty, Graph Theory with Applications (Macmillan Press Ltd, London, 1976).

[2] P. Erdős and R.K. Guy, Crossing number problems, Amer. Math. Monthly 80 (1973) 52-58.

doi: $10.2307 / 2319261$

[3] M.R. Garey and D.S. Johnson, Crossing number is NP-complete, SIAM J. Algebraic Discrete Methods 4 (1983) 312-316.

doi: $10.1137 / 0604033$

[4] V.R. Kulli and M.H. Muddebihal, Characterization of join graphs with crossing number zero, Far East J. Appl. Math. 5 (2001) 87-97.

[5] D.J. Kleitman, The crossing number of $K_{5, n}$, J. Combin. Theory Ser. B 9 (1970) 315-323. doi:10.1016/S0021-9800(70)80087-4

[6] M. Klešč, The join of graphs and crossing numbers, Electron. Notes Discrete Math. 28 (2007) 349-355.

doi:10.1016/j.endm.2007.01.049

[7] L. Tang, J. Wang and Y.Q. Huang, The crossing number of the join of $C_{m}$ and $P_{n}$, Internat. J. Math. Com. 1 (2007) 110-116.

[8] M. Klešč and S. Schrötter, The crossing numbers of join products of paths with graphs of order four, Discuss. Math. Graph Theory 31 (2011) 321-331. doi:10.7151/dmgt.1548

[9] M. Klešč, The crossing numbers of join of the special graph on six vertice with path and cycle, Discrete Math. 310 (2010) 1475-1481.

doi:10.1016/j.disc.2009.08.018 
[10] M. Klešč and S. Schrötter, The crossing numbers of join of paths and cycles with two graphs of order five, Lecture Notes in Comput. Sci. 7125 (2012) 160-167. doi:10.1007/978-3-642-28212-6_15

[11] Y.H. Peng and Y.C. Yiew, The crossing number of $P(3,1) \times P_{n}$, Discrete Math. 306 (2006) 1941-1946.

doi:10.1016/j.disc.2006.03.058

Received 17 May 2016

Revised 14 November 2016

Accepted 14 November 2016 